\title{
Gasping as a predictor of short- and long-term outcomes in patients with cardiac arrest: a systematic review and meta-analysis
}

\author{
Qin-Yue Guo ${ }^{1,2, *}$, Jing Xu' ${ }^{2,3}$, Qin-Dong Shi ${ }^{1}$
}

\author{
${ }^{1}$ Department of Critical Care Medicine, \\ The First Affiliated Hospital of Xi'an \\ Jiaotong University, 277 Yanta West \\ Street, Xi'an, 710061 Shaanxi, P. R. China \\ ${ }^{2}$ Weil Institute of Emergency and Critical \\ Care Research, Virginia Commonwealth \\ University, Richmond, VA, USA \\ ${ }^{3}$ Department of Emergency Medicine, \\ The First Affiliated Hospital of Xi'an \\ Jiaotong University, 277 Yanta West \\ Street, Xi'an, 710061 Shaanxi, P. R. China
}

*Correspondence

guoqinyue@163.com

(Qin-Yue Guo)

\begin{abstract}
Aims: Cardiac arrest (CA), a worldwide health problem in the public, has a high mortality rate. While gasping has been associated with increased survival and Return of Spontaneous Circulation (ROSC) after cardiac arrest in some studies, more data is needed.

Data sources: The literature on cardiac arrest and gasping from Medline, PubMed, Embase, Cochrane, and Web of Science up to September 2020 were searched, categorized, and analyzed. The association of CA and gasping outcomes were assessed by risk ratios (RR) and $95 \%$ confidence intervals (CI). Heterogeneity, sensitivity analysis, and publication bias was discussed.

Results: 8 studies from 7 articles were included. A fixed-effect or random-effect model was applied to all the outcomes using estimate pooled risk ratios (RR). Patients suffering gasping during CA were 2.08 times more likely to have return of spontaneous ROSC compared with patients without gasping $(\mathrm{RR}=2.08,95 \% \mathrm{CI}$ : $1.93-2.25, P<0.001)$. There was a significant increase in the survival to discharge rate of the patients who gasped $(\mathrm{RR}=3.75,95 \% \mathrm{CI}=3.27-4.29, P<0.001)$; along with a more favorable neurological 1-year survival ( $\mathrm{RR}=8.02,95 \% \mathrm{CI}: 5.56,11.57 ; P<0.001)$ and the presence of a shockable cardiac rhythm $(\mathrm{RR}=2.82,95 \% \mathrm{CI}$ : $2.50,3.18 ; P<0.001)$.

Conclusions: The presence of agonal respirations is positively associated with ROSC, achieving a shockable cardiac rhythm, increasing survival rate to discharge, and a neurologically favorable 1-year survival. Gasping may play an important role in cardiopulmonary resuscitation (CPR) training.
\end{abstract}

\section{Keywords}

Gasping; Agonal respiration; Cardiac arrest; Cardiopulmonary resuscitation (CPR)

\section{Introduction}

Cardiac arrest is a worldwide public health problem, and is also a leading cause of death. Over 800,000 Europeans and North Americans suffer an out-of-hospital cardiac arrest (OHCA) every year, which is associated with high mortality [1]. Although significant efforts have been made, the outcomes are still poor with an overall survival averaging only $7.6 \%$ for the last 30 years $[2,3]$. Post-resuscitation cerebral and myocardial dysfunction are the main causes of death following OHCA [4].

While several factors had been proven to affect the short and long-term outcomes of OHCA, such as the initial rhythm and the interval time to defibrillation, there is a growing interest in spontaneous respiratory activity during cardiac arrest or resuscitation. This gasping phenomenon has been associated with a greater chance of achieving Return of Spontaneous Circulation (ROSC), thus increasing the survival rate to discharge following OHCA, and preserving cerebral function after cardiopulmonary resuscitation (CPR) [5, 6].
Various terms have been used to describe this spontaneous respiratory activity, such as agonal breathing, agonal respirations, and abnormal breathing. Gasping has been defined by the Glossary Committee of the International Union of Physiological Sciences in 1973 as an abrupt, sudden and transient inspiratory effort [7]. Gasping after the onset of cardiac arrest (CA) has been reported to be associated with improved survival in animal models of cardiac arrest in previous studies [8-13]. A possible mechanism for the improved survival rate may be related to increased cardiac output and cerebral perfusion $[10,14]$.

While the observational short-term outcomes of ROSC and survival to discharge had been well studied in patients with gasping following OHCA, long-term outcomes such as cerebral function is still limited [15]. More in-depth analysis is needed to demonstrate the association of gasping and improved outcomes in OHCA patients.

A better knowledge of gasping is critical for both clinical 
care and out-of-hospital CPR since it provides a better understanding of the physiology of cardiac arrest. The aim of this study is to further clarify and update the association of gasping associated with short and long-term outcomes following OHCA.

\section{Methods}

\subsection{Search strategy}

The literature of cardiac arrest and gasping from PubMed, Embase, Medline, Cochrane, and Web of Science up to September 2020 were searched, categorized, and analyzed with the search terms "cardiac arrest", "CPR", "heart arrest", "out-ofhospital", and "gasping", "agonal breathing", "agonal respiration", "abnormal breathing”. In this analysis, only articles published in English were included.

\subsection{Selection criteria}

The primary analysis of randomized trials or secondary analysis of observational cohort studies were eligible for inclusion by comparing clinical outcomes between patients with and without gasping during cardiac arrest. All the studies were selected by two independent reviewers based on the following criteria: (a) the subjects were human adults; (b) eligible clinical outcomes include ROSC, survival to discharge, survival to one-month, or Cerebral Performance Category (CPC) score; (c) sufficient published data for estimating the risk ratio (RR) with $95 \%$ CI. Studies were excluded if there was no sufficient data for pooling.

\subsection{Quality assessment}

The quality of a selected study was assessed by using a semiquantification principle star system, consists of eight items in three forums including crowd selection, comparability, exposure assessment, and outcome evaluation utilizing the Newcastle-Ottawa scale [16].

\subsection{Data extraction}

Data was extracted by two independent reviewers from each eligible study. ROSC was the primary outcome variable, while survival to hospital discharge, survival to one-month, and oneyear survival with Cerebral Performance Category (CPC) score $\leq 2$ were the secondary outcome variables.

The study characteristics included the first author's names, publication years, countries, type of recordings, study designs, and the total number of patients who suffered CA.

\subsection{Statistical analysis}

All analyses were conducted with Review Manager (version 5.3). Risk ratios (RR) and 95\% CIs were calculated to determine the association of gasping and CA. The pooled RR and $95 \%$ CI were calculated by fixed-effect or random-effect model. If there was significant heterogeneity $\left(P<0.05, I^{2}\right.$ $>50 \%$ ), a random-effects model was adopted; otherwise, fixed-effects models were applied if there was no significant heterogeneity $\left(P \geq 0.05, I^{2} \leq 50 \%\right.$ ). Heterogeneity was tested by the Q-test based on the Chi-square or $I^{2}$ statistic test. Whenever heterogeneity was present, sensitivity analyses were performed by the absence of one trial in each turn to test the influence of a single study on the overall pooled estimate. Publication bias was evaluated by visually inspecting funnel plots.

\section{Results}

\subsection{Study selection}

Initially, the keywords search returned 407 records, of which 225 duplicated studies and 64 studies that referred to animals were excluded. After scanning the abstracts of the remaining potential eligible studies, 12 reviews, 5 case reports, and 83 irrelevant subjects of gasping and outcomes in patients with CA were further excluded. After full-text articles were assessed for eligibility, 11 studies were excluded, 5 of which failed to provide enough data and 6 reported no outcomes of interest. Finally, 8 studies from 7 articles [5, 6, 17-21], which involved patients who suffered a cardiac arrest, qualified for the inclusion criteria in the meta-analysis to estimate pooled data. Marterns et al. [18] reported two different databases (1983-1987 (1) and 1991-1993 (2)). The flow of studies, including the reasons for inclusion and exclusion of studies, is shown in Fig. 1.

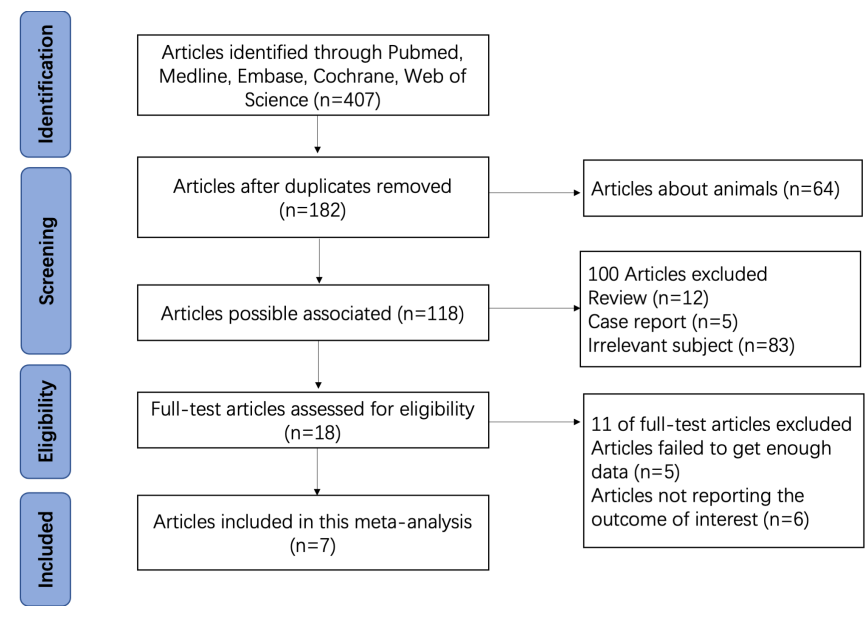

F I G U R E 1. Selection of studies for the meta-analysis.

\subsection{Study characteristic and validity assessment}

The characteristics of the studies are shown in Table 1. All seven studies were observational, which included prospective [19-21] and retrospective cohort studies. Only one study [20] involved an in-hospital cardiac arrest, the others were outof-hospital cardiac arrest. Among the seven cohorts, four studies from three articles reported the ROSC rate of gasping compared with no gasping patients, while six studies from five articles reported survival to hospital discharge, three studies reported favorable neurological outcomes, and four studies reported the relationship between gasping and cardiac rhythm during cardiac arrest. The quality assessments of the seven studies are shown in Table 2. 
TA B L E 1. Study characteristics.

\begin{tabular}{|c|c|c|c|c|c|c|c|c|}
\hline Authors & Year & Country & Study design & $\begin{array}{c}\text { Study } \\
\text { Population }\end{array}$ & $\begin{array}{l}\text { Type } \\
\text { patients }\end{array}$ & $\begin{array}{l}\text { Totally Study } \\
\text { Participants }\end{array}$ & Location & Outcome of interests \\
\hline Bobrow & 2008 & USA & $\begin{array}{l}\text { Retrospective } \\
\text { cohort }\end{array}$ & Adult & Non-trauma & 1218 & $\begin{array}{l}\text { Out-of- } \\
\text { hospital }\end{array}$ & $\begin{array}{l}\text { Survival to discharge, } \\
\text { Shockable rhythm }\end{array}$ \\
\hline Clark & 1992 & USA & $\begin{array}{l}\text { Retrospective } \\
\text { cohort }\end{array}$ & Adult, Infant & Non-trauma & 445 & $\begin{array}{l}\text { Out-of- } \\
\text { hospital }\end{array}$ & Survival to discharge \\
\hline Martens & 1995 & Belgium & $\begin{array}{l}\text { Retrospective } \\
\text { cohort }\end{array}$ & Adult, Infant & $\begin{array}{l}\text { Trauma, Non- } \\
\text { trauma }\end{array}$ & $\begin{array}{l}\text { (1) } 3083 ;(2) \\
2127\end{array}$ & $\begin{array}{l}\text { Out-of- } \\
\text { hospital }\end{array}$ & ROSC, Survival to discharge \\
\hline Takei & 2017 & Japan & $\begin{array}{l}\text { Retrospective } \\
\text { cohort }\end{array}$ & Adult, Infant & $\begin{array}{l}\text { Trauma, Non- } \\
\text { trauma }\end{array}$ & 9374 & $\begin{array}{l}\text { Out-of- } \\
\text { hospital }\end{array}$ & $\begin{array}{l}\text { Neurologically favorable } \\
1 \text { year survival, Shockable } \\
\text { rhythm }\end{array}$ \\
\hline Inaba & 2015 & Japan & $\begin{array}{l}\text { Prospective } \\
\text { cohort }\end{array}$ & Adult, Infant & $\begin{array}{l}\text { Trauma, Non- } \\
\text { trauma }\end{array}$ & 7048 & $\begin{array}{l}\text { Out-of- } \\
\text { hospital }\end{array}$ & $\begin{array}{l}\text { Neurologically favorable } \\
1 \text { year survival, Shockable } \\
\text { rhythm }\end{array}$ \\
\hline Marengo & 2018 & Switzerla & $\begin{array}{l}\text { Prospective } \\
\text { Cohort }\end{array}$ & Adult, Infant & $\begin{array}{l}\text { Trauma, Non- } \\
\text { trauma }\end{array}$ & 270 & In Hospital & $\begin{array}{lr}\text { ROSC; } & \text { Survival to } \\
\text { discharge, } & \text { Shockable } \\
\text { rhythm } & \end{array}$ \\
\hline Debaty & 2017 & France & $\begin{array}{l}\text { Prospective } \\
\text { Cohort }\end{array}$ & Adult & Non-trauma & 1888 & $\begin{array}{l}\text { Out-of- } \\
\text { hospital }\end{array}$ & $\begin{array}{l}\text { ROSC; Survival to } \\
\text { discharge, Neurologically } \\
\text { favorable } 1 \text { year survival, } \\
\text { Shockable rhythm }\end{array}$ \\
\hline
\end{tabular}

TA B L E 2. Assessment of the validity appraisal of the included seven studies.

\begin{tabular}{|c|c|c|c|c|c|c|c|}
\hline & Bobrow & Clark & Martens & Takei & Inaba $\mathrm{I}$ & Marengo & Debaty \\
\hline Representativeness of the exposed cohort & $*$ & $*$ & $*$ & $*$ & $*$ & $*$ & $*$ \\
\hline Selection of the non-exposed cohort & $*$ & $*$ & $*$ & $*$ & $*$ & $*$ & $*$ \\
\hline Ascertainment of exposure & $*$ & * & $*$ & $*$ & $*$ & * & $*$ \\
\hline Demonstration that outcome of interest was not present at start of study & $*$ & * & $*$ & $*$ & $*$ & $*$ & $*$ \\
\hline Comparability of cohorts on the basis of the design or analysis & $* *$ & - & $* *$ & $* *$ & $* *$ & ** & ** \\
\hline Assessment of outcome & * & * & * & * & * & * & $*$ \\
\hline Was follow-up long enough for outcomes to occur & $*$ & $*$ & $*$ & $*$ & $*$ & * & $*$ \\
\hline Adequacy of follow up of cohorts & $*$ & $*$ & $*$ & - & - & - & $*$ \\
\hline
\end{tabular}

\subsection{Meta-analysis}

\subsubsection{ROSC}

Four studies in three articles estimated the association of gasping with ROSC in patients suffering a cardiac arrest (Fig. 2). When low heterogeneity across the studies $\left(\mathrm{Chi}^{2}=5.97, \mathrm{df}=\right.$ $\left.3, P=0.11, I^{2}=50.0 \%\right)$ was obtained, a fixed-effect model was applied to estimate a pooled RR of 2.08 (95\% CI: 1.932.25), indicating that patients who suffered a cardiac arrest with gasping were 2.08 times more likely to develop ROSC compared to those without gasping. A funnel plot was utilized to assess the publication bias of all the studies (Fig. 3). We excluding a single study each time for sensitivity analysis. The result did not change the statistical outcome when the studies were excluded.

\subsection{Survival to discharge}

In six studies involving 8746 patients after cardiac arrest, 331 out of 1464 patients $(22.6 \%)$ survived to hospital discharge in the gasping group, while 432 out of 7282 patients $(5.9 \%)$ in no gasping group survived to discharge. In view of the very low heterogeneity among these studies $\left(\mathrm{Chi}^{2}=3.23, \mathrm{df}=5, P\right.$ $=0.66, I^{2}=0 \%$ ) the fixed-effects model was then adopted. A pooled analysis indicated that there was a significant difference of survival to discharge between the patients who gasped during cardiac arrest compared with those who did not (RR $=3.75,95 \% \mathrm{CI}=3.27-4.29, P<0.001$ ) (Fig. 2). As shown in Fig. 3, a funnel plot was used to assess the publication bias of all studies; the points representing the evaluated studies in the funnel plot were concentrated at the top, indicating that the studies had high precision and large sample sizes. A sensitivity analysis was performed by the same method above which excluding every single study each time and the results did not change the outcome statistically by excluding any studies, indicating that the outcome showed a high stability. 


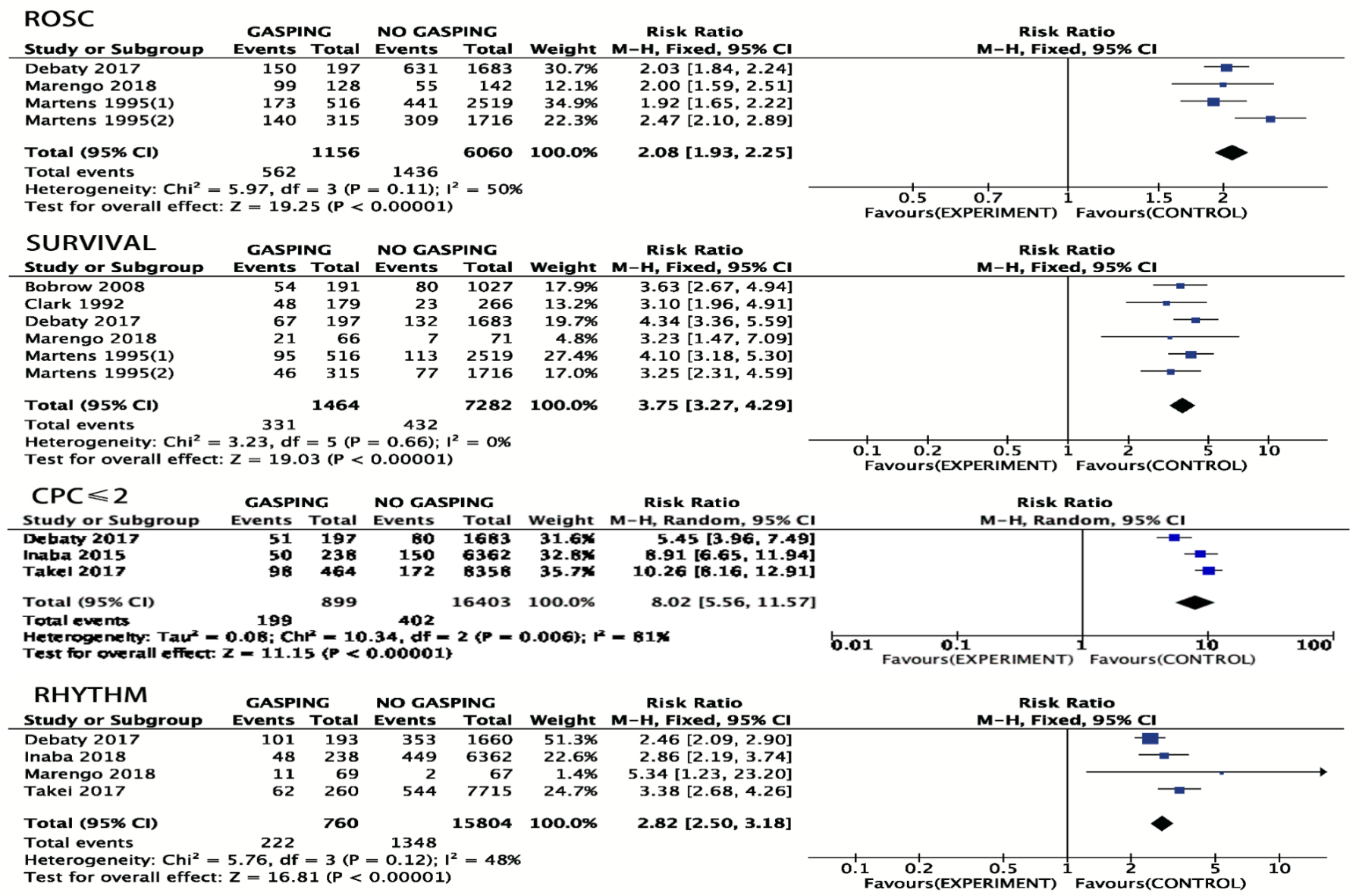

F I G U R E 2. Forrest plot showing the pooled estimate of the effect between gasping and no gasping. OR, risk ratio; CI, confidence limit; M-H: Mantel Haenszel method.

\section{$3.5 \mathrm{CPC} \leq 2$}

Only three studies showed outcomes on the association of gasping with neurologically favorable one-year survival. These cohort studies were included to estimate the pooled RR of 8.02 (95\% CI: 5.56, 11.57; $P<0.001)$ (Fig. 2). Although the pooled result of the trials showed a significantly increased chance to achieve a good neurological outcome when patients gasped during cardiac arrest, the association of gasping and $\mathrm{CPC} \leq 2$ was highly heterogeneous $\left(\mathrm{Chi}^{2}=10.34 ; \mathrm{df}=2 ; P\right.$ $\left.=0.006 ; I^{2}=81 \%\right)$. The funnel plots depicted in Fig. 3 were generally asymmetrical, indicating the presence of publication bias. Although only three observational studies were involved in this analysis, sensitivity analysis demonstrated that the absence of any study each time did not influence the outcome of the statistics, indicating that the outcome was stable.

\subsection{Shockable rhythm}

Meta-analysis of four studies showed a significant increase in a shockable cardiac rhythm during cardiac arrest in gasping patients compared with patients with no gasping. A fixedeffect model estimated the pooled RR as 2.82 (95\% CI: 2.50 , 3.18; $P<0.001)$ with low heterogeneity $\left(\mathrm{Chi}^{2}=5.76\right.$; $\mathrm{df}=$ 3; $P=0.12 ; I^{2}=48 \%$ ) (Fig. 2). The patients were much more likely to have a shockable cardiac rhythm during cardiac arrest who were gasped. A funnel plot was used to assess the publication bias of all studies. The funnel plot (Fig. 3) was almost symmetrical, indicating that publication bias may exist but was not apparent. A sensitivity analysis was performed as the same method above. The results showed a decreased heterogeneity $\left(I^{2}=95 \%\right.$ to $\left.I^{2}=48 \%\right)$ and higher RR ( 2.04 to 2.82) when excluding the study of Bobrow2018 [6]. Since the data from the Bobrow2018 study only referred to ventricular fibrillation (VF) but not a totally shockable rhythm, we deleted the data from this study. However, the absence of any study data did not change the outcome statistically since the affected quantity was small.

\section{Discussion}

In this meta-analysis, we reviewed the studies regarding the outcomes of patients gasping during CPR. The pooled results suggested that the present of gasping significantly improve the short- and long-term outcomes in cardiac arrest patients.

Despite the widespread use of automated external defibrillators (AED), survival to hospital discharge remains poor following OHCA [2]. Several methods to assess neurological prognosis in patients after cardiac arrest who are successfully resuscitated include neurological examination, EEG, biomarkers and neuroimaging studies [22]. However, none of the above modalities has shown to predict neurological recovery. Therefore, other parameters are required for assessment of the long-term neurological function after CA. Results from a survey of survivors of OHCA in Japan showed that 30-day neu- 

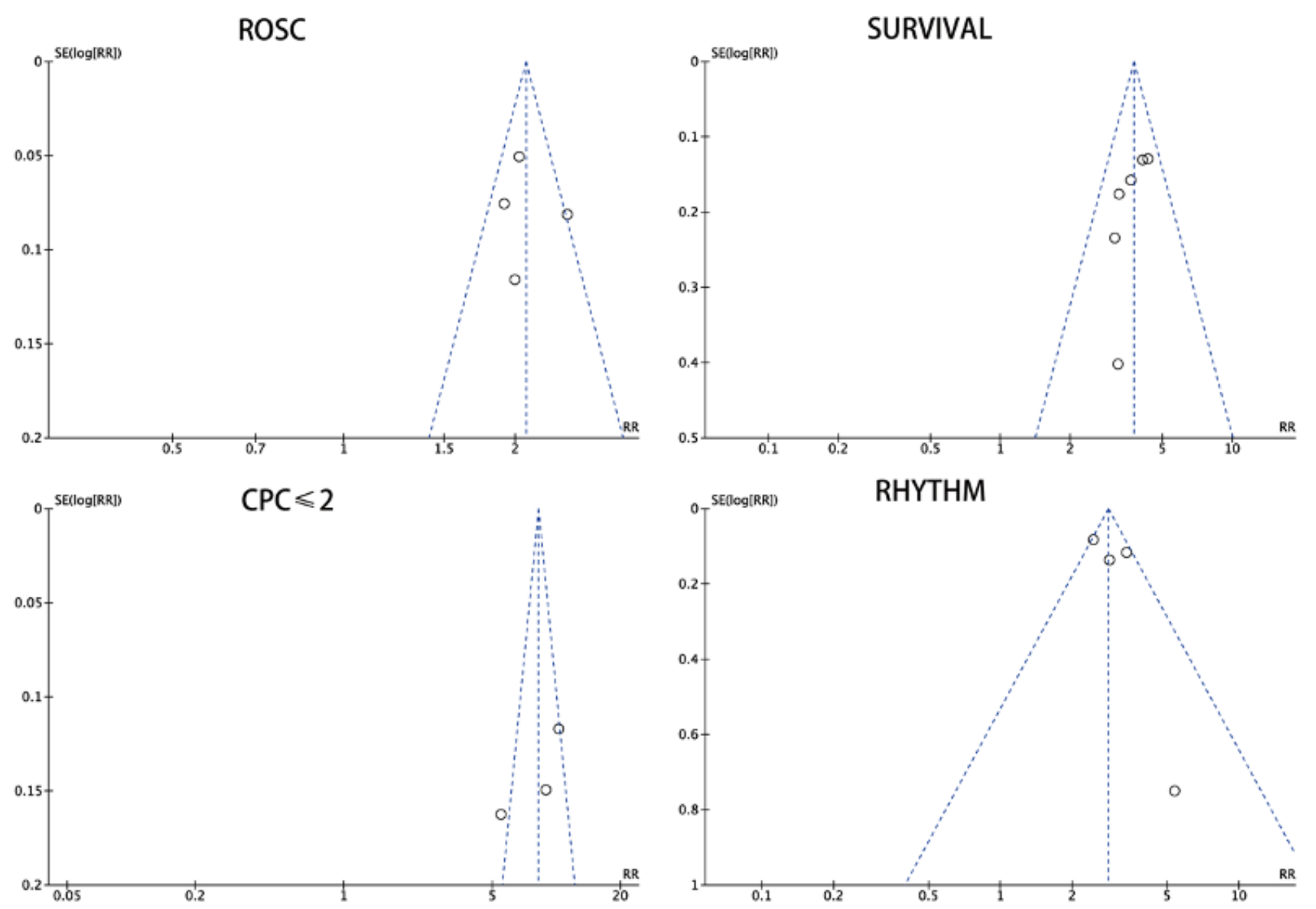

F I G U R E 3. Funnel plot of pooling of gasping effect on different outcome. The hollow dots and dotted line indicate individual studies and $95 \%$ confidence intervals, respectively.

rologic outcomes were more favorable in patients who gasped [23], suggesting that gasping may be a more reliable tool to predict neurologic outcomes in these patients. Clarification of the relationship between gasping and prognosis during or after CPR, will allow us to better understand the underlining mechanisms of CPR.

Favorable initial rhythms, such as ventricular tachycardia (VT) and ventricular fibrillation (VF), are well-known as factors associated with decreased mortality. Nearly thirty years ago, the presence of gasping during CPR was found to be more likely associated with a shockable cardiac rhythm and improved hospital survival to discharge [5]. Subsequent studies found that gasping during cardiopulmonary resuscitation was common in both animals and humans $[6,13]$. The incidence of gasping before the arrival of emergency medical personal has been shown to be 55\% [5]. Our previous studies in animals also demonstrated that the gasping during cardiac arrest exactly improved cardiac output, maintained more optimal amplitudes of VF waveforms, enhanced cerebral blood flow, and decreased intracranial pressure (ICP) [10, 24]. The study by Debaty2017 [21] found that gasping during CPR was a "natural biomarker for the presence of brainstem activity", and strongly demonstrated its association with better long-term survival with good brain function.

Researchers have previously shown that gasping is generated by the intrinsic neuronal activities located in the medulla oblongata [25-27]. Gasping has been demonstrated to be an auto-resuscitative phenomenon in human infants [28]. A gasping-like behavior is almost always present in terminally ill adult patients dying from a variety of causes.

It is important to recognize that gasping, has been mistaken for life signs that may result in the abandonment of attempts for resuscitation by a bystander in some, but not all studies $[29,30]$. The ability to recognize gasping improves the diagnostic accuracy of a OHCA and should be included in all cardiopulmonary resuscitation training programs.

Our findings demonstrated that patients who suffered gasping during cardiac arrest were more likely to achieve ROSC, and significantly increased the survival to discharge with no heterogeneity. Moreover, gasping significantly increased the chance for the presence of a shockable cardiac rhythm during cardiac arrest and is a favorable factor for a better neurological one-year survival.

There are some limitations with our study. Randomized control trails (RCT) studies were not included in this metaanalysis, since only observational studies have been reported for OHCA. Only three studies were qualified for reporting neurological outcomes, leading to a highly heterogeneous group of patients. Most of the studies provided no details about the frequency of gasping, the depth of gasping or the timing of gasping in relation to OHCA.

In conclusion, we found that the presence of agonal respirations is positively associated with achievement of ROSC, shockable cardiac rhythms, increased survival to discharge, 
and neurologically favorable one-year survival. Therefore, gasping needs be emphasized in CPR training, and should be recognized by bystanders or medical workers as soon as possible, to achieve the most favorable outcomes following OHCA.

\section{AUTHOR CONTRIBUTIONS}

Qinyue Guo conceived and designed the study; Qinyue Guo and Jing $\mathrm{Xu}$ had full access to all the data in the study and take responsibility for the integrity of the data and the accuracy of the data analysis. Qinyue Guo drafted the manuscript and made the statistical analysis, Qindong Shi revised it, all authors critically reviewed the manuscript.

\section{ACKNOWLEDGMENT}

Financial supported by Natural Science Research Plan of Shaanxi Province (S2018-JC-YB-1708).

\section{CONFLICT OF INTEREST}

There are no conflicts of interest to disclose for all authors of this study.

\section{REFERENCES}

[1] Nichol G, Thomas E, Callaway CW, Hedges J, Powell JL, Aufderheide $\mathrm{TP}$, et al. Regional variation in out-of-hospital cardiac arrest incidence and outcome. Journal of the American Medical Association. 2008; 300: 1423-1431.

[2] Sasson C, Rogers MAM, Dahl J, Kellermann AL. Predictors of survival from out-of-hospital cardiac arrest: a systematic review and metaanalysis. Circulation: Cardiovascular Quality and Outcomes. 2010; 3: 63-81.

[3] Benjamin EJ, Blaha MJ, Chiuve SE, Cushman M, Das SR, Deo R, et al. Heart disease and stroke statistics-2017 update: a report from the american heart association. Circulation. 2017; 135: e146-e603.

[4] Dell'anna AM, Scolletta S, Donadello K, Taccone FS. Early neuroprotection after cardiac arrest. Current Opinion in Critical Care. 2014; 20 : 250-258.

[5] Clark JJ, Larsen MP, Culley LL, Graves JR, Eisenberg MS. Incidence of agonal respirations in sudden cardiac arrest. Annals of Emergency Medicine. 1992; 21: 1464-1467.

[6] Bobrow BJ, Zuercher M, Ewy GA, Clark L, Chikani V, Donahue D, et al. Gasping during cardiac arrest in humans is frequent and associated with improved survival. Circulation. 2008; 118: 2550-2554.

[7] Glossary on respiration and gas exchange. Journal of Applied Physiology. 1973; 34: 549-558.

[8] Yang L, Weil MH, Noc M, Tang W, Turner T, Gazmuri RJ. Spontaneous gasping increases the ability to resuscitate during experimental cardiopulmonary resuscitation. Critical Care Medicine. 1994; 22: 879-883.

[9] Yang L, Weil MH, Noc M, Turner T, Tang W. Spontaneous gasping during cardiac arrest. Critical Care Medicine. 1994; 22: A136.

[10] Xie J, Weil MH, Sun S, Yu T, Tang W. Spontaneous gasping generates cardiac output during cardiac arrest. Critical Care Medicine. 2004; 32: 238-240.

[11] Ristagno G, Tang W, Sun S, Weil MH. Spontaneous gasping produces carotid blood flow during untreated cardiac arrest. Resuscitation. 2007; 75: 366-371.

[12] Menegazzi JJ, Check BD. Spontaneous agonal respiration in a swine model of out-of-hospital cardiac arrest. Academic Emergency Medicine. 1995; 2: 1053-1056.
[13] Zuercher M, Ewy GA, Otto CW, Hilwig RW, Bobrow BJ, Clark L, et al. Gasping in response to basic resuscitation efforts: observation in a swine model of cardiac arrest. Critical Care Research and Practice. 2010; 2010: 351638.

[14] Srinivasan V, Nadkarni VM, Yannopoulos D, Marino BS, Sigurdsson G, McKnite $\mathrm{SH}$, et al. Spontaneous gasping decreases intracranial pressure and improves cerebral perfusion in a pig model of ventricular fibrillation. Resuscitation. 2006; 69: 329-334.

[15] Wolfskeil M, Vanwulpen M, Duchatelet C, Monsieurs KG, HachimiIdrissi S. Detection and quantification of gasping during resuscitation for out-of-hospital cardiac arrest. Resuscitation. 2017; 117: 40-45.

[16] Wells G, Shea B, O'Connell D, Peterson J, Welch V, Losos M, et al. The Newcastle-Ottawa Scale (NOS) for assessing the quality of nonrandomized studies in meta-analysis. Ottawa Health Research Institute. 2012.

[17] Takei Y, Tanaka Y, Maeda T, Ohta K, Inaba H. Agonal breathing without carotid pulse on EMS arrival but not agonal breathing with carotid pulse is associated with better outcomes of out-of-hospital respiratory and cardiac arrests. Resuscitation. 2017; 118: e70.

[18] Martens P, Mullie A, Vanhaute O. Clinical status before and during cardiopulmonary resuscitation versus outcome in two consecutive databases. European Journal of Emergency Medicine. 1995; 2: 17-23.

[19] Inaba H, Takei Y, Kamikura T, Funada A, Wato Y, Sakagami S, et al. Agonal respiration with and without carotid pulsation on emergency medical technician's arrival at patient. Circulation. 2015; 132: A14300.

[20] Marengo L, Ummenhofer W, Pascal G, Harm F, Lüthy M, Zürcher M. Abstract 17847: gasping is a valid predictor of ROSC and hospital discharge for in-hospital cardiac arrest occurring on the ward. Circulation. 2015; 132: A17847.

[21] Debaty G, Labarere J, Frascone RJ, Wayne MA, Swor RA, Mahoney $\mathrm{BD}$, et al. Long-term prognostic value of gasping during out-of-hospital cardiac arrest. Journal of the American College of Cardiology. 2017; 70 : 1467-1476.

[22] Grundmann S, Busch HJ. Neurologic prognosis after cardiac arrest. The New England Journal of Medicine. 2009; 361: 1999.

[23] SOS-KANTO study group. Cardiopulmonary resuscitation by bystanders with chest compression only (SOS-KANTO): an observational study. The Lancet. 2007; 369: 920-926.

[24] Pernat A, Weil M, Bisera J, Tang W, Fukui M. Spontaneous gasping maintains more optimal amplitudes of ventricular fibrillation waveforms during cardiac arrest. USA: Lippincott Williams \& Wilkins 227 East Washington SQ, Philadelphia, PA. 1998.

[25] Gozal D, Torres JE, Gozal YM, Nuckton TJ. Characterization and developmental aspects of anoxia-induced gasping in the rat. Neonatology. 1996; 70: 280-288.

[26] St.-John WM, Paton JFR. Role of pontile mechanisms in the neurogenesis of eupnea. Respiratory Physiology \& Neurobiology. 2004; 143: 321-332.

[27] St-John WM, Paton JFR. Respiratory-modulated neuronal activities of the rostral medulla which may generate gasping. Respiratory Physiology \& Neurobiology. 2003; 135: 97-101.

[28] Sridhar R, Thach BT, Kelly DH, Henslee JA. Characterization of successful and failed autoresuscitation in human infants, including those dying of SIDS. Pediatric Pulmonology. 2003; 36: 113-122.

[29] Franek O, Sukopova P. Laypersons are not able to recognize cardiac arrest when "gasping" is present. Resuscitation. 2008; 77: S46-S47.

[30] Chęciński I, Zyśko D, Smereka J, Gajek J, Mirecka-Świerzko J, Ściborski $\mathrm{R}$, et al. The presence of agonal respiration during cardiac arrest and resuscitation attempts by witnesses. Clinical and Experimental Medicine. 2011; 20: 761-765.

How to cite this article: Qin-Yue Guo, Jing $\mathrm{Xu}$, Qin-Dong Shi. Gasping as a predictor of short- and long-term outcomes in patients with cardiac arrest: a systematic review and meta-analysis. Signa Vitae. 2021;17(2):208-213. doi:10.22514/sv.2021.006. 